\title{
Effect of bovine lactoferrin and human lactoferrin on the proliferative activity of the osteoblast cell line MC3T3-E1 in vitro
}

\author{
J. L. Zhang, ${ }^{*}$ X. Han,${ }^{* 1}$ Y. J. Shan, ${ }^{*}$ L. W. Zhang, ${ }^{*}{ }^{1}{ }^{1}$ M. Du, ${ }^{*}$ M. Liu, ${ }^{*}$ H. X. Yi, $†$ and Y. Ma \\ ${ }^{*}$ School of Chemistry and Chemical Engineering, Harbin Institute of Technology, Harbin, 150090, China \\ †College of Food Science and Engineering, Ocean University of China, Qingdao, 266003, China
}

\begin{abstract}
We conducted a comparative in vitro study on the proliferative effects of natural human lactoferrin (nhLF) and bovine lactoferrin (bLF) on osteoblasts. We investigated cell proliferation, cell survival, cell cycle, and mRNA and protein expression of proliferating cell nuclear antigen. Results indicated that treatment with $100 \mu \mathrm{g} / \mathrm{mL}$ of bLF or nhLF promoted the proliferation and sustenance of osteoblasts, and increased the length of the $\mathrm{G}_{2} / \mathrm{M}$ and $\mathrm{S}$ phases compared with the untreated osteoblasts. Results of real-time quantitative PCR and Western blot showed that mRNA and protein expression of proliferating cell nuclear antigen by osteoblasts treated with bLF or nhLF were greater than those of the untreated control. At the same concentration, bLF demonstrated a greater effect on osteoblast proliferation than did nhLF. This study provides insights of significance in the utlization of bLF in healthy food formulas.
\end{abstract}

Key words: natural human lactoferrin, bovine lactoferrin, proliferation effect, osteoblast

\section{INTRODUCTION}

Lactoferrin ( $\mathbf{L F}$ ) is an $80-\mathrm{kDa}$ iron-binding glycoprotein belonging to the transferrin family, and it is produced by mammal epithelial cells of exocrine glands, including human, cow, sheep, and horse (García-Montoya et al., 2012). As a pleiotropic factor, it is involved in many biological processes such as regulation of the levels of free iron in body fluids and immunoregulation, and it is known to have antitumor, antimicrobial, and antivirus activities (Naot et al., 2005). Studies have implicated bovine LF (bLF) as a growth factor that promotes mitosis of osteoblasts, stimulates proliferation and maturation of primary osteoblasts, accelerates the

Received May 13, 2017.

Accepted November 10, 2017

${ }^{1}$ Corresponding authors: xhan@hit.edu.cn and zhanglw@hit.edu.cn differentiation of osteoblasts, and protects osteoblasts apoptosis from serum withdrawal (Cornish et al., 2004; Balasubramanian et al., 2012; García-Montoya et al., 2012).

The use of bLF in foods has been regarded as safe by the scientific community. According to the European Food Safety Agency (EFSA), the maximum allowed inclusion of bLF is $1,000 \mathrm{mg} / \mathrm{L}$ in infant formulas, $3,000 \mathrm{mg} / 100 \mathrm{~g}$ in chewing gums, and $133 \mathrm{mg} / 100 \mathrm{~g}$ in dairy products (EFSA, 2012). In the United States, the approved bLF range is 13 to $100 \mathrm{mg} / 100 \mathrm{~mL}$ for infant formula, $100 \mathrm{mg} / 100 \mathrm{~g}$ for yogurt, $400 \mathrm{mg} / 100 \mathrm{~g}$ for powdered milk, $200 \mathrm{mg} / 100 \mathrm{~g}$ for milk dessert and sugars, and $30 \mathrm{mg} / \mathrm{g}$ for sweets (FDA, 2011).

Natural human lactoferrin (nhLF) is an ideal food supplement for infants; however, little human lactoferrin is available for use in the industry; thus hindering its use compared with bLF, which is readily available in animals. Both bLF and recombinant human lactoferrin (rhLF) have been studied with respect to their effects on osteoblasts. The proliferative effect of rhLF on rat osteoblast-like cells was reported to be less than that of bLF (Grey et al., 2004). However, few studies have investigated nhLF with respect to proliferative effects on osteoblasts.

Several studies have indicated that bLF, nhLF, and rhLF cannot substitute for one another because of marked differences: nhLF and bLF consist of 691 and 689 AA, respectively, with $69 \%$ homology and similar 3-dimensional structures (Lorget et al., 2002). In vitro and in vivo investigations have demonstrated differences in their antivirus and cancer prevention mechanisms (Arnold et al., 2002; Tsuda et al., 2002). Additionally, rhLF contains more mannose residues in its polysaccharide chains than nhLF, especially when produced using a fungal express system. Consequently, rhLF has a shorter half-life than nhLF, which is thought to be related to the mannose content (van Veen et al., 2004; Kruzel et al., 2013). Other differences between rhLF and nhLF include composition of polysaccharide chains, digestive stability, and antimicrobial activity (van Veen et al., 2004; Kruzel et al., 2013). In addition, 
diverse $\mathrm{N}$-glycan structures (fucosylated and sialylated) in bLF, nhLF, and rhLF have been reported, although rhLF $N$-glycans are more similar to those in bLF than to those in nhLF (Le Parc et al., 2017).

Our understanding of the proliferation effect of nhLF on osteoblasts is currently limited. Therefore, the objective of this study was to investigate the proliferative effects of bLF and nhLF on osteoblasts to improve the utilization of bLF in healthy food formulations.

\section{MATERIALS AND METHODS}

\section{Reagents and Materials}

The bLF used in this experiment was donated by Kanpure Ltd. Co. (Harbin, Heilongjiang, China). 3-(4,5-Dimethylthiazol-2-yl)-2,5-diphenyltetrazolium bromide (MTT) and nhLF were purchased from Sigma-Aldrich (St. Louis, MO). Cell counting kit-8 (CCK-8) was supplied by WST (Dojinbo, Japan); $\alpha$-minimal essential medium ( $\alpha$-MEM), trypsin-EDTA, and penicillin-streptomycin were all purchased from Hyclone (Logan, UT); and fetal bovine serum was from Gibco (Grand Island, NY). Primary proliferating cell nuclear antigen (PCNA; rabbit monoclonal) antibody was from Abcam (Cambridge, MA), and the primary GADPH (polyclonal) antibody was prepared by Bioworld (St. Louis, MO). Horseradish peroxidase (HRP)goat anti-rabbit IgG secondary antibody was made by KPL (Milford, MA). Solarbio (Beijing, China) provided the enhanced HRP-DAB (3,3'-diaminobenzidine) chromogenic substrate kit. The polyvinylidene fluoride (PVDF) membrane was purchased from Millipore (Danvers, MA). All-in-One qPCR Mix was purchased from GeneCopoeia (San Diego, CA).

\section{Cell Culture and Assays}

The MC3T3-E1 subclone 14 osteoblast cell line was purchased from the cell library of the Chinese Academy of Sciences (Shanghai, China). The cells were cultured in $\alpha$-MEM medium with $10 \%$ fetal bovine serum and $1 \%$ penicillin-streptomycin under a humidified atmosphere consisting of $5 \% \mathrm{CO}_{2}$ and $95 \%$ air at $37^{\circ} \mathrm{C}$ (Steri-cycle $\mathrm{CO}_{2}$ incubator, Thermo Fisher Scientific Inc., Waltham, MA), and the medium was changed every 2 to 3 d. For subcultures, cells at $90 \%$ confluence were passaged after detachment in $0.25 \%$ trypsin. For assays, the cell number was counted by using a hemocytometer and adjusted to a cell density of $1 \times 10^{5}$ cells/ $\mathrm{mL}$. For the proliferation and survival assays, $100 \mu \mathrm{L}$ of cells was seeded into 96-well plates and sextuplicates conducted at each concentration. For the cell cycle, real-time quantitative PCR, and Western blot assays, 7 $\mathrm{mL}$ of cells was seeded into $90-\mathrm{mm}$ plates, with triplicates conducted at each concentration. After overnight attachment, the medium with experimental compounds $(1,10$, and $100 \mu \mathrm{g} / \mathrm{mL}$ bLF or nhLF) was refreshed and cells incubated again. The incubation periods used in the proliferation, survival, and cell cycle assays were 24 , 48, and $72 \mathrm{~h}$, whereas those for PCR and Western blot assays were $48 \mathrm{~h}$.

\section{Iron Saturation of bLF and nhLF Analysis}

Ten milliliters of solution of bLF or nhLF (2 mg/ $\mathrm{mL}$ ) was digested in nitric acid and the resulting residue was transferred into a $10-\mathrm{mL}$ volumetric flask and made up to $10 \mathrm{~mL}$ with deionized water. The iron concentration was determined with the aid of an optical emission spectrometer (Optima 5300 DV, PerkinElmer, Waltham, MA). Iron saturation was calculated according to the following formula:

$$
X 1=\frac{\left(C-C_{0}\right) \times V \times f}{m \times 1,000},
$$

where $X 1$ is the iron mass fraction of sample $(\mathrm{mg} / \mathrm{g})$; $C$ is the iron concentration of sample $(\mathrm{mg} / \mathrm{L}) ; C_{0}$ is the iron concentration of control $(\mathrm{mg} / \mathrm{L}) ; V$ is the volume of sample $(\mathrm{mL}) ; f$ is the dilution ratio; and $m$ is the mass of sample $(\mathrm{g})$.

Then, iron saturation was calculated as follows:

$$
X=\frac{X 1}{1.4} \times 100 \%,
$$

where $X$ is the iron saturation of sample (\%) and 1.4 is the correction factor, which was derived as follows:

$$
C F=\frac{M W I \times n F I}{R L F},
$$

where $C F$ is the correction factor; $M W I$ is the molecular weight of iron (55.85); $n F I$ is the number of molecules of ferric iron (2); $R L F$ is the relative molecular mass of LF $(80,000)$; and multiplied by 1,000 to convert grams to milligrams.

\section{Proliferative Activity of bLF and nhLF on MC3T3-E1}

Proliferative activity of bLF or nhLF on osteoblasts was measured according to the method of Hou et al. (2014). After incubation, $10 \mu \mathrm{L}$ of $\mathrm{MTT}(5 \mathrm{mg} / \mathrm{mL})$ 
was added to each well and further incubated in the dark for $4 \mathrm{~h}$ at $37^{\circ} \mathrm{C}$. The solution of each well was removed and $150 \mu \mathrm{L}$ of dimethyl sulfoxide was added to dissolve the blue formazan crystals, which was followed by shaking at room temperature for $10 \mathrm{~min}$. The absorbance was read at $490 \mathrm{~nm}$ using a microplate reader (BioTek-Eon, Winooski, VT).

\section{Survival Assay of bLF- and nhLF-Treated MC3T3-E1}

Cell survival was assessed using the CCK-8 assay according to the method of Ju et al. (2015). After incubation, $10 \mu \mathrm{L}$ of CCK-8 was added to each well and further incubated in the dark for $4 \mathrm{~h}$. The absorbance was read at $450 \mathrm{~nm}$ using a microplate reader.

\section{Cell Cycle of bLF- and nhLF-Treated MC3T3-E1}

The cell cycle was determined by a flow cytometer (BD Bioscience, San Jose, CA) according to the method of Miao et al. (2015). Cells from each plate were harvested and transferred to new tubes, which were then centrifuged at $100 \times g$ for $5 \mathrm{~min}$. The supernatant was carefully removed and $1 \mathrm{~mL}$ of buffer solution was added to each tube to resuspend cells overnight at $4^{\circ} \mathrm{C}$. Propidium iodide solution (BD Bioscience) was added to each tube and the contents were filtered through a 50- $\mu \mathrm{m}$ nylon mesh, after which they were analyzed using a flow cytometer.

\section{RNA Isolation and Real-Time Quantitative PCR}

Total cellular RNA was purified using a GeneMark kit (GMbiolab Co., Ltd., Taiwan), and cDNA was synthesized with a GeneCopoeia kit. Independent reaction mixtures were performed using the same cDNA for both the target gene and internal control. After an initial denaturation $\left(95^{\circ} \mathrm{C}\right.$ for $\left.10 \mathrm{~min}\right), 40$ amplification cycles were performed, with each cycle including denaturation at $95^{\circ} \mathrm{C}$ for $10 \mathrm{~s}$, annealing at $57^{\circ} \mathrm{C}$ for $20 \mathrm{~s}$, and extension at $72^{\circ} \mathrm{C}$ for $30 \mathrm{~s}$ using a 7300 Real Time PCR System (Applied Biosystems, Hercules, CA). Relative levels of gene expression were calculated by the $2^{-\Delta \Delta \mathrm{CT}}$ method (where $\mathrm{CT}=$ cycle threshold). Oligonucleotide primers used for amplification are presented in Table 1.

\section{Protein Extraction and Western Blot Analysis}

Total cellular protein was extracted by using radioimmuno-precipitation assay (RIPA) buffer (Solarbio, Beijing, China), and the concentrations of lysates were detected by the bicinchoninic acid (BCA) assay (BCA Protein Assay kit; Thermo Fisher Scientific). The concentration of all lysates was adjusted to be the same according to the results of the BCA assay. Protein from each sample was loaded onto a $12 \%$ SDS-PAGE gel and semi-dry transferred (Trans-Blot SD, Bio-Rad Laboratories, Hercules, CA) onto the PVDF membranes for immune blot analysis. The PVDF membranes were blocked with $3 \%$ BSA at room temperature for $1 \mathrm{~h}$, followed by the addition of primary antibodies and further incubated overnight at $4^{\circ} \mathrm{C}$. The membranes were washed with $1 \times$ Tris-buffered saline (TBS)Tween (twice) and $1 \times$ TBS (once) after which they were incubated with secondary antibody for $1 \mathrm{~h}$. The membranes were washed using the above-mentioned washing sequence and $2 \mathrm{~mL}$ of $\mathrm{DAB}$ was added to each membrane. To check the equal loading of the proteins, GADPH served as the internal control. Band area intensity was quantified using the Quantity One software from Bio-Rad Laboratories.

\section{Statistical Analysis}

Experimental data in triplicates or sextuplicates were analyzed using the Statistical Package for the Social Sciences v. 20.0 (SPSS/IBM Corp., Chicago, IL). A one-way ANOVA was used to compare mean values using a significance level of $P<0.05$. The differences among the mean values within groups and their classification were achieved using Tukey's post hoc test.

\section{RESULTS}

\section{Iron Saturation of bLF and $n h L F$}

To determine whether iron saturations of bLF and nhLF reflected the nonmodified (native) state, iron concentrations of bLF and nhLF were measured using an optical emission spectrometer and their iron saturations calculated according to the formulas above. The iron mass fraction and iron saturation of bLF were

Table 1. Oligonucleotide primer sequences for target genes used in real-time quantitative PCR

\begin{tabular}{llc}
\hline Gene of interest & Primer sequence $\left(5^{\prime}-3^{\prime}\right)$ & Annealing temperature $\left({ }^{\circ} \mathrm{C}\right)$ \\
\hline PCNA & Forward: GAAGGCTTCGACACATACCGC & 60 \\
RAPDH & $\begin{array}{l}\text { Reverse: CAGCTGTACTCCTGTTCTGGG } \\
\text { Reverse: CGAACCCTAAGGCCAACC }\end{array}$ & 60 \\
\hline
\end{tabular}


$0.2508 \mathrm{mg} / \mathrm{g}$ and $17.91 \%$, respectively, and those of nhLF were $0.1551 \mathrm{mg} / \mathrm{g}$ and $11.08 \%$, respectively.

\section{Effects of bLF and nhLF on MC3T3-E1 Proliferation}

The results of proliferative effects of bLF and nhLF on MC3T3-E1 are presented in Figure 1. We observed a linear increase in proliferation of MC3T3-E1 cells with increasing bLF and nhLF concentrations and incubation periods. At $72 \mathrm{~h}, 100 \mu \mathrm{g} / \mathrm{mL}$ bLF exhibited the greatest effect in enhancing proliferation of cells compared with the control or the other bLF treatments (1 and $10 \mu \mathrm{g} / \mathrm{mL}$ bLF; $P<0.05)$. At $24 \mathrm{~h}$, the 1 and 10 $\mu \mathrm{g} / \mathrm{mL}$ bLF treatments did not differ significantly from the control. No significant difference was found between the $1 \mu \mathrm{g} / \mathrm{mL}$ bLF treatment and the control at either 48 or $72 \mathrm{~h}$.

Similar to bLF, $100 \mu \mathrm{g} / \mathrm{mL}$ nhLF resulted in greater cell production compared with the control or the other 2 treatments $(1$ and $10 \mu \mathrm{g} / \mathrm{mL}$ nhLF; $P<0.05)$. At 72 $\mathrm{h}$, the differences in the proliferation effect of 10 and $100 \mu \mathrm{g} / \mathrm{mL}$ nhLF were significant $(P<0.05)$, but no difference was observed between $1 \mu \mathrm{g} / \mathrm{mL}$ nhLF and the control. At $24 \mathrm{~h}$, no significant differences were observed between any of the nhLF treatments and the control. Furthermore, at $48 \mathrm{~h}, 100 \mu \mathrm{g} / \mathrm{mL}$ nhLF had a significantly greater effect than the control $(P<0.05)$.

\section{Effect of bLF and nhLF on MC3T3-E1 Viability}

The effects of bLF and nhLF on the cell viability are shown in Figure 2. The cell numbers increased after $24 \mathrm{~h}$ and cell mortality began at $48 \mathrm{~h}$ in both bLF and nhLF treatments. At $72 \mathrm{~h}$, cell numbers decreased $36.65 \%$ with the $100 \mu \mathrm{g} / \mathrm{mL}$ bLF treatment and $42.56 \%$ with the $100 \mu \mathrm{g} / \mathrm{mL} \mathrm{hLF}$ treatment. The cell numbers of bLF and hLF treatments were 45.65 and $32.73 \%$ higher than that of the control $(P<0.05)$. Regardless of the treatment timepoint, the cell numbers remained higher with increasing treatment concentrations for both bLF and $\mathrm{nhLF}$. The $100 \mu \mathrm{g} / \mathrm{mL}$ bLF treatment resulted in a significantly higher $(P<0.05)$ survival rate than other treatments. The 1 and $10 \mu \mathrm{g} / \mathrm{mL}$ bLF treatments were not significantly different from each other.

\section{Effect of bLF and nhLF on Cell Cycle of MC3T3-E1}

The effects of bLF and nhLF treatments on the cell cycle are presented in Table 2. The maximum effect on cell cycle was observed after $48 \mathrm{~h}$ of treatment by $100 \mu \mathrm{g} / \mathrm{mL}$ bLF or nhLF. The $\mathrm{G}_{0} / \mathrm{G}_{1}$ phase of cells treated with $100 \mu \mathrm{g} / \mathrm{mL}$ bLF was $73.90 \%$, which was significantly shorter $(P<0.05)$ than that of the control (87.03\%). Meanwhile, the $\mathrm{G}_{2} / \mathrm{M}$ and $\mathrm{S}$ phases were 8.09

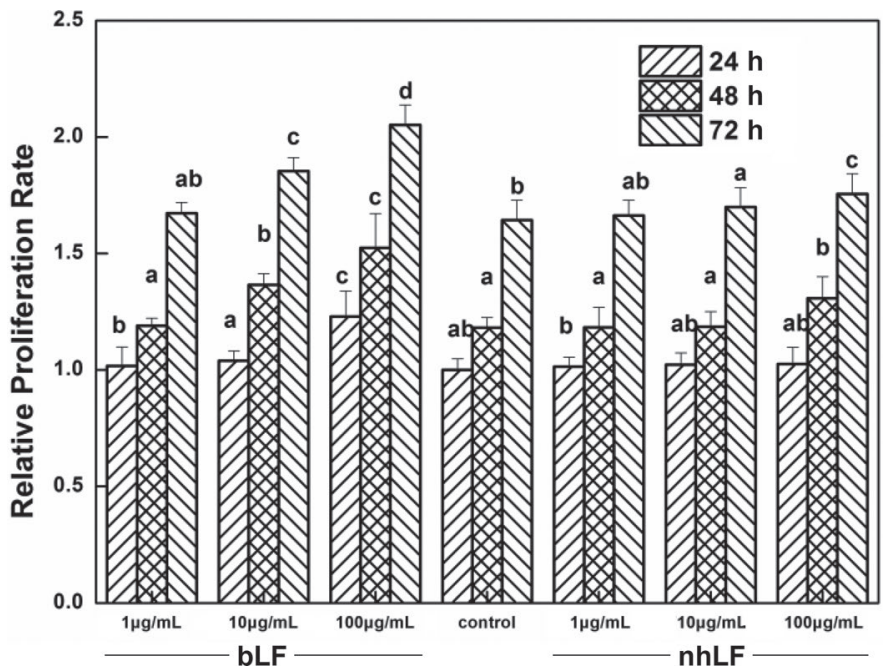

Figure 1. Effects of different concentrations of bovine lactoferrin (bLF) and natural human lactoferrin (nhLF) on osteoblast proliferation. The cell number of control at $24 \mathrm{~h}$ was normalized to a value of 1 . Different letters between groups across all treatments and timepoints represent significant differences $(P<0.05)$. Error bars indicate SD.

and $18.29 \%$, which were significantly longer $(P<0.05)$ than those of the control (7.05 and 5.39\%).

Similar to bLF, $100 \mu \mathrm{g} / \mathrm{mL}$ nhLF resulted in a better effect than the other nhLF concentrations on shortening the cell cycle: the $\mathrm{G}_{0} / \mathrm{G}_{1}$ phase decreased $(76.34 \%)$, and the $\mathrm{G}_{2} / \mathrm{M}$ and $\mathrm{S}$ phases increased $(7.87$ and $15.33 \%$, respectively). However, bLF performed better than nhLF $(P<0.05)$.

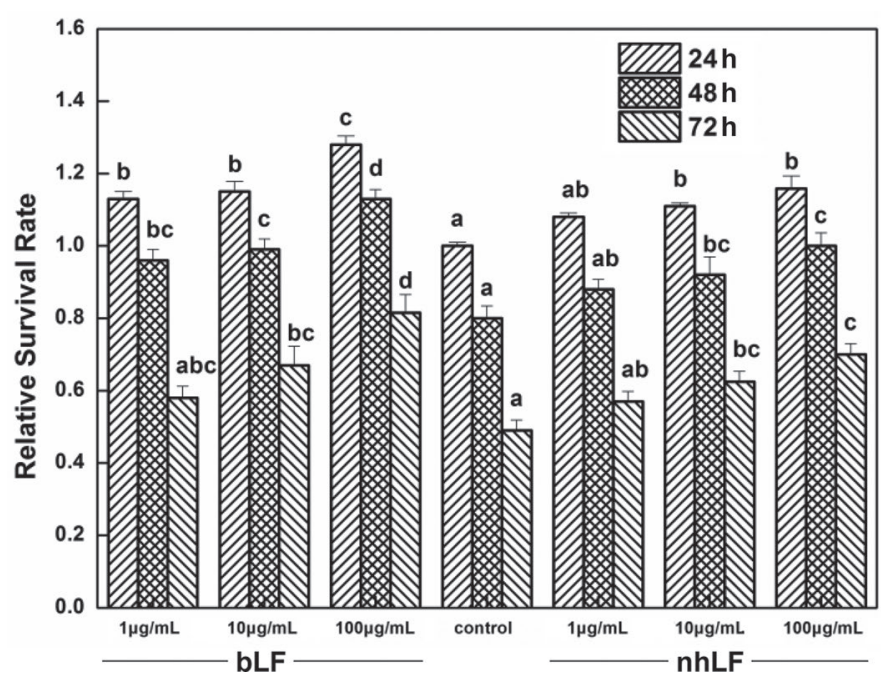

Figure 2. Effects of different concentrations of bovine lactoferrin (bLF) and natural human lactoferrin (nhLF) on cell viability (survival). The cell number of control at $24 \mathrm{~h}$ was normalized to a value of 1 . Different letters between groups across all treatments and timepoints represent significant differences $(P<0.05)$. Error bars indicate SD. 

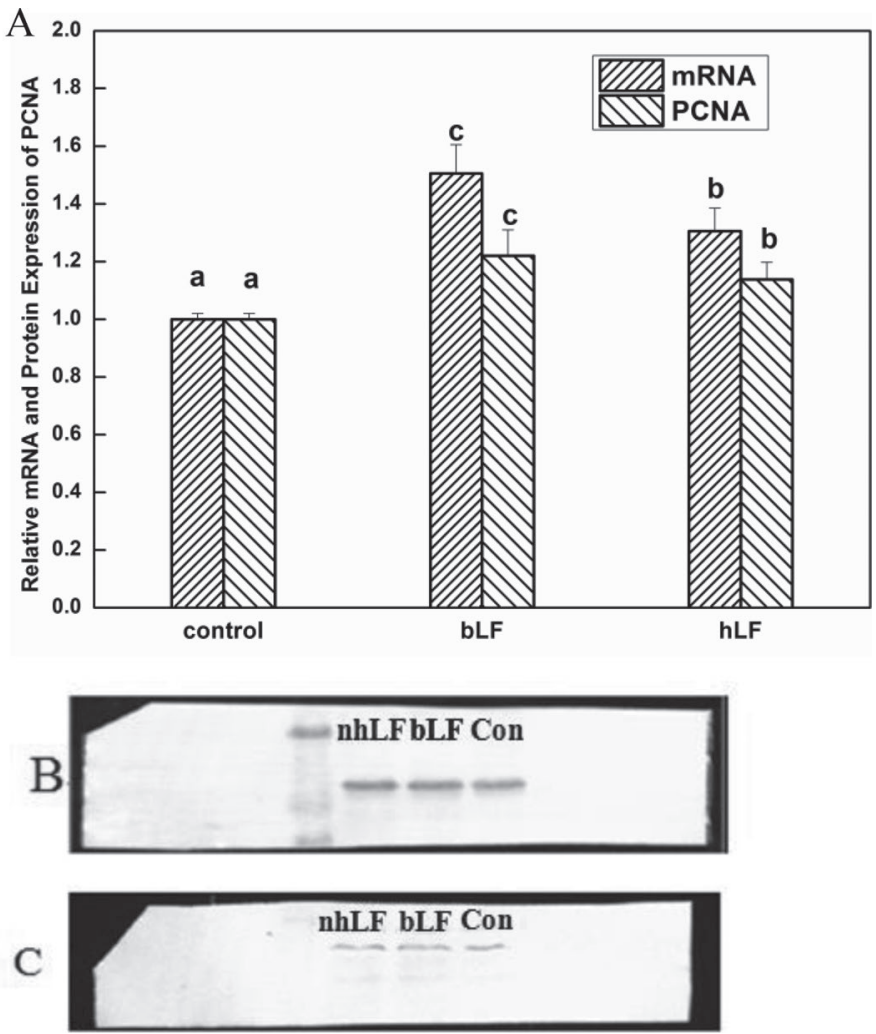

Figure 3. Expression of mRNA and protein of proliferating cell nuclear antigen (PCNA) treated with bovine lactoferrin (bLF) and natural human lactoferrin (nhLF). Expression levels of PCNA in the control (medium only) were normalized to a value of 1 . Different letters between treatments within mRNA or protein (PCNA) expression represent significant differences $(P<0.05)$. Error bars indicate SD. (A) Fold change in mRNA and protein levels of PCNA; (B) internal control (GAPDH expression) in osteoblasts treated with nhLF, bLF and control; and (C) PCNA expression in osteoblasts treated with nhLF, bLF, and control.

\section{Changes in mRNA and Protein Expression of PCNA}

The mRNA and protein expression of PCNA are shown in Figure 3. The bLF treatment resulted in 66.00 and $20.00 \%$ increases in mRNA and protein expression of PCNA, respectively, compared with the control $(P<0.05)$. Likewise, 28.00 and $10.40 \%$ increases in mRNA and protein expression, respectively, of PCNA was noted after treatment with nhLF compared with the control $(P<0.05)$. It is important to note that increases in mRNA and protein expression of PCNA were 27.45 and $16.82 \%$ higher, respectively, with bLF than nhLF $(P<0.05)$.

\section{DISCUSSION}

In our study, the iron saturations of bLF and nhLF were about 18 and $11 \%$, respectively. This is in line with a study that reported an iron saturation range of 15 to $20 \%$ for bLF and about $10 \%$ for nhLF (Gibbons et al., 2015). Hence, both LF in our study retained their native states. It has been demonstrated that LF at its physiological concentration promotes the proliferation and accelerates the differentiation of osteoblasts in vitro (Naot et al., 2011). Both bLF and nhLF showed time- and dose-dependent relationships on the proliferation of osteoblasts. The $1 \mu \mathrm{g} / \mathrm{mL}$ bLF treatment had no significant effect on proliferation compared with the control. However, the 10 and $100 \mu \mathrm{g} / \mathrm{mL}$ bLF treatments increased proliferation significantly. The same pattern was seen with the nhLF treatments. The proliferation effect of bLF and rhLF on osteoblasts was compared with the results of other studies (Cornish et al., 2004), which reported that the proliferation effect with $10 \mu \mathrm{g} / \mathrm{mL}$ of bLF was 2 times higher than that with $10 \mu \mathrm{g} / \mathrm{mL}$ of rhLF. Our results indicated that the proliferation effect of $10 \mu \mathrm{g} / \mathrm{mL}$ bLF was 1.17 times

Table 2. Effect of different concentrations of bovine lactoferrin (bLF) and natural human lactoferrin (nhLF) on the percentage of total cell cycle time $( \pm \mathrm{SD})$ in each phase of the osteoblast cell line MC3T3-E1

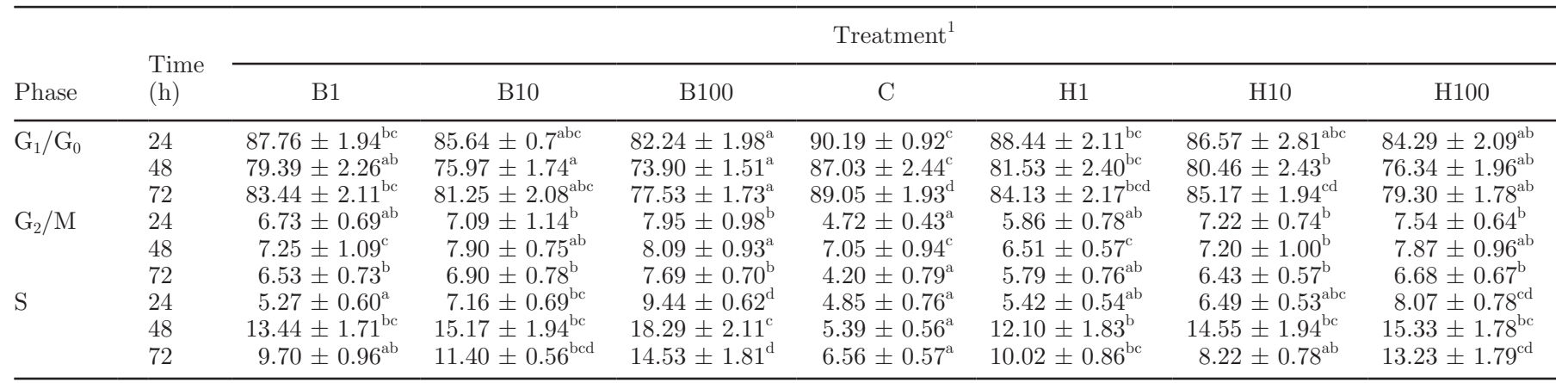

\footnotetext{
${ }^{\mathrm{a}-\mathrm{d}}$ Means with different letters between groups are significantly different $(P<0.05)$.

${ }^{1}$ Treatments B1, B10, and B100 = 1, 10, and $100 \mu \mathrm{g} / \mathrm{mL}$ of bLF, respectively; C represents control; H1, H10, and H100 represent 1, 10 , and 100 $\mu \mathrm{g} / \mathrm{mL}$ of $\mathrm{nhLF}$, respectively.
} 
higher than that of $10 \mu \mathrm{g} / \mathrm{mL} \operatorname{nhLF}(P<0.05)$. Thus, the proliferation effect of nhLF appears to be better than that of rhLF, even though a direct comparison has not been made. The results of the study also demonstrated that the proliferation effect improved as the dose increased (to $100 \mu \mathrm{g} / \mathrm{mL}$ of bLF or rhLF; Cornish et al., 2004). This was in line with our findings in which proliferation occurred in a dose-dependent manner. However, the proliferation effect decreased when the concentration reached $1,000 \mu \mathrm{g} / \mathrm{mL} \mathrm{bLF}$ and there was no significant difference compared with $10 \mu \mathrm{g} / \mathrm{mL}$ bLF (Cornish et al., 2004). The $100 \mu \mathrm{g} / \mathrm{mL}$ bLF treatment performed significantly better than either $10 \mu \mathrm{g} / \mathrm{mL}$ or $1,000 \mu \mathrm{g} / \mathrm{mL}$ bLF (Cornish et al., 2004; Grey et al., 2004). Overall, in the current study, bLF showed better proliferation effects on osteoblasts than nhLF at the same concentration and the same treatment time.

Cornish and coworkers (2006) demonstrated that iron saturation had no significant effect on proliferation when osteoblasts were treated with $1 \mu \mathrm{g} / \mathrm{mL} \mathrm{bLF}$; however, at 10 and $100 \mu \mathrm{g} / \mathrm{mL} \mathrm{bLF}$, iron saturation affected the proliferation of the osteoblast significantly. Their study also indicated that LF with a lower iron saturation had a greater proliferation effect than LF with a higher iron saturation, and that a higher iron saturation reduced cell proliferation (Cornish et al., 2006). In the current study, the iron saturation of bLF was $18 \%$, which was higher than that of nhLF (11\%); thus, we presume that the proliferation effect of bLF on osteoblasts should be lower than that of nhLF. However, our findings revealed otherwise. Although the reason for this phenomenon is not clear, the differences in polysaccharides chains between bLF and nhLF could be a plausible explanation. This assumption is due to the fact that polysaccharide chain composition has been reported to have an effect on digestive stability and antimicrobial activity of LF (van Veen et al., 2004; Kruzel et al., 2013). Although the reason for this phenomenon is not clear, difference in polysaccharide chains between bLF and bhLF could be a plausible explanation. The polysaccharides could affect the digestive stability and antimicrobial activity, and may affect the cell proliferation. . Research has demonstrated that increases in tryptophan and $\alpha$-helix content and a decrease in $\beta$-structure content were ensured by an increased iron saturation of LF. The same study indicated that the osteogenic activity of LF decreased with increasing iron saturation (Wang et al., 2013).

Besides iron saturation, other factors may affect cell proliferation. It is well documented that LF reduces apoptosis in osteoblasts and thus is an effective mechanism for cell survival because LF increases the expression of IGF-1 to exert anti-apoptosis activity. Both bLF and nhLF had higher anti-apoptosis activity than the control. Lactoferrin also stimulated the proliferation of osteoblasts by shortening the cell cycle. In the current study, a flow cytometer was used to detect changes in the cell cycle of osteoblasts treated with bLF and nhLF. Both bLF and nhLF shortened the cell cycle in a dose-dependent manner, which was accompanied by a remarkable increase in length of the cell cycle of both the $\mathrm{S}$ phase and the $\mathrm{G}_{2} / \mathrm{M}$ phase. The $\mathrm{G}_{0} / \mathrm{G}_{1}$ phase was $3.20 \%$ shorter after bLF treatment at $100 \mu \mathrm{g} / \mathrm{mL}$ than after nhLF at the same dose. The bLF treatments of $\mathrm{G}_{2} / \mathrm{M}$ and $\mathrm{S}$ phases at that same dose were 5.44 and $19.31 \%$ longer than that of nhLF with the same dose. This suggests that bLF was more effective at shortening the cell cycle than nhLF. The S phase was the most upregulated phase by bLF treatment, and the speed of cell division was accelerated.

It is well known that LF has DNA-binding properties, acting as an enzyme to catalyze some reactions to accelerate the expression of mRNA (García-Montoya et al., 2012). One study demonstrated that LF upregulated intestinal mRNA expression in postnatal piglets and decreased expression of the luxS gene in Streptococcus pneumoniae (Kasala et al., 2015). Proliferating cell nuclear antigen plays an important role in cell proliferation and is a good indicator of cell proliferation (Zhao et al., 2013), and its induction stimulates the proliferation of vascular smooth muscle cells (Kulldorff et al., 2000). In addition, PCNA is expressed during the late $\mathrm{G}_{0} / \mathrm{G}_{1}$ phase, $\mathrm{S}$ phase, and early $\mathrm{G}_{2} / \mathrm{M}$ phase (Nakajima et al., 2011). In this study, we found that bLF and nhLF upregulated the mRNA expression of PCNA and lengthened the $\mathrm{S}$ and $\mathrm{G}_{2} / \mathrm{M}$ phases. Considering both these results, we speculate that LF could promote cell proliferation by simultaneously regulating PCNA expression and the cell cycle.

\section{CONCLUSIONS}

This study evaluated the effects of bLF and nhLF on cell proliferation, cell survival, cell cycle, and mRNA and protein expression of PCNA. Both bLF and nhLF significantly influenced these factors compared with the control. However, bLF had a greater proliferative effect on osteoblasts than did nhLF. Further studies should investigate the role of polysaccharide chains in the proliferation activity of bLF and nhLF and the utilization of bLF in the food industry.

\section{ACKNOWLEDGMENTS}

This work was financially supported by National Natural Science Foundation of China (31771987), Heilongjiang Key Science and Technology Plan (GA16B201-2). 


\section{REFERENCES}

Arnold, D., A. M. Di Biase, M. Marchetti, A. Pietrantoni, P. Valenti, L. Seganti, and F. Superti. 2002. Antiadenovirus activity of milk proteins: Lactoferrin prevents viral infection. Antiviral Res. 53:153-158.

Balasubramanian, S. A., D. C. Pye, and M. D. P. Willcox. 2012. Levels of lactoferrin, secretory IgA and serum albumin in the tear film of people with keratoconus. Exp. Eye Res. 96:132-137.

Cornish, J., K. Palmano, K. E. Callon, M. Watson, J. M. Lin, P. Valenti, D. Naot, A. B. Grey, and I. R. Reid. 2006. Lactoferrin and bone; structure-activity relationships. Biochem. Cell Biol. 84:297-302.

Cornish, J., K. E. Callon, D. Naot, K. P. Palmano, T. Banovic, U. Bava, M. Watson, J.-M. Lin, P. Tong, and Q. Chen. 2004. Lactoferrin is a potent regulator of bone cell activity and increases bone formation in vivo. Endocrinology 145:4366-4374.

EFSA. 2012. Panel on Dietetic Products, Nutrition and Allergies (NDA): Scientific Opinion on bovine lactoferrin. EFSA J. 10:28112824.

FDA. 2011. Generally Recognized As Safe (GRAS) Notification 000423 for cow's milk-derived lactoferrin as a component of cow's milk-based infant formulas, cow's milk products, and chewing gum. https:// www.fda.gov/downloads/Food/IngredientsPackagingLabeling/ GRAS/NoticeInventory/ucm303340.pdf.

García-Montoya, I. A., T. S. Cendon, S. Arevalo-Gallegos, and Q. Rascon-Cruz. 2012. Lactoferrin a multiple bioactive protein: An overview. Biochim. Biophys. Acta 1820:226-236.

Gibbons, J. A., J. R. Kanwar, and R. K. Kanwar. 2015. Iron-free and iron-saturated bovine lactoferrin inhibit survivin expression and differentially modulate apoptosis in breast cancer. BMC Cancer 15:425-440.

Grey, A., T. Banovic, Q. Zhu, M. Watson, K. Callon, K. Palmano, J. Ross, D. Naot, I. R. Reid, and J. Cornish. 2004. The low-density lipoprotein receptor-related protein 1 is a mitogenic receptor for lactoferrin in osteoblastic cells. Mol. Endocrinol. 18:2268-2278.

Hou, J. M., M. Wu, Q. M. Lin, F. Lin, Y. Xue, X. H. Lan, E. Y. Chen, M. L. Wang, H. Y. Yang, and F. X. Wang. 2014. Lactoferrin promote primary rat osteoblast proliferation and differentiation via up-regulation of insulin-like growth factor-1 expression. Mol. Biol. Rep. 41:5019-5030.

Ju, W., S. Li, Z. Wang, Y. Liu, and D. Wang. 2015. Decorin protects human hepatoma HepG2 cells against oxygen-glucose deprivation via modulating autophagy. Int. J. Clin. Exp. Med. 8:13347-13352.

Kasala, E. R., L. N. Bodduluru, R. M. Madana, V. Ak, R. Gogoi, and C. C. Barua. 2015. Chemopreventive and therapeutic potential of chrysin in cancer: Mechanistic perspectives. Toxicol. Lett. 233:214-225.
Kruzel, M. L., J. K. Actor, M. Zimecki, J. Wise, P. Płoszaj, S. Mirza, M. Kruzel, S.-A. Hwang, X. Ba, and I. Boldogh. 2013. Novel recombinant human lactoferrin: Differential activation of oxidative stress related gene expression. J. Biotechnol. 168:666-675.

Kulldorff, M., L. M. Mcshane, A. Schatzkin, L. S. Freedman, M. J. Wargovich, C. Woods, M. Purewal, R. W. Burt, M. Lawson, and D. J. Mateski. 2000. Measuring cell proliferation in the rectal mucosa comparing bromodeoxyuridine (BrdU) and proliferating cell nuclear antigen (PCNA) assays. J. Clin. Epidemiol. 53:875-883.

Le Parc, A., S. Karav, C. Rouquié, E. A. Maga, A. Bunyatratchata, and D. Barile. 2017. Characterization of recombinant human lactoferrin $N$-glycans expressed in the milk of transgenic cows. PLoS One 12:e0171477.

Lorget, F., J. Clough, M. Oliveira, M.-C. Daury, A. Sabokbar, and E. Offord. 2002. Lactoferrin reduces in vitro osteoclast differentiation and resorbing activity. Biochem. Biophys. Res. Commun. 296:261-266

Miao, X. D., L. Cao, Q. Zhang, X. Y. Hu, and Y. Zhang. 2015. Effect of PI3K-mediated autophagy in human osteosarcoma MG63 cells on sensitivity to the chemotherapy drug cisplatin. Asian Pac. J. Trop. Med. 8:731-738.

Nakajima, K., Y. Kanno, M. Nakamura, X. D. Gao, A. Kawamura, F Itoh, and A. Ishisaki. 2011. Bovine milk lactoferrin induces synthesis of the angiogenic factors VEGF and FGF2 in osteoblasts via the p44/p42 MAP kinase pathway. Biometals 24:847-856.

Naot, D., A. Chhana, B. G. Matthews, K. E. Callon, P. C. Tong, J.-M. Lin, J. L. Costa, M. Watson, A. B. Grey, and J. Cornish. 2011. Molecular mechanisms involved in the mitogenic effect of lactoferrin in osteoblasts. Bone 49:217-224.

Naot, D., A. Grey, I. R. Reid, and J. Cornish. 2005. Lactoferrin-A novel bone growth factor. Clin. Med. Res. 3:93-101.

Tsuda, H., K. Sekine, K.-I. Fujita, and M. Iigo. 2002. Cancer prevention by bovine lactoferrin and underlying mechanisms: A review of experimental and clinical studies. Biochem. Cell Biol. 80:131-136.

van Veen, H. A., M. E. Geerts, P. H. van Berkel, and J. H. Nuijens. 2004. The role of N-linked glycosylation in the protection of human and bovine lactoferrin against tryptic proteolysis. Eur. J. Biochem. 271:678-684.

Wang, X. Y., H. Y. Guo, W. Zhang, P. C. Wen, H. Zhang, Z. R. Guo, and F. Z. Ren. 2013. Effect of iron saturation level of lactoferrin on osteogenic activity in vitro and in vivo. J. Dairy Sci. 96:33-39.

Zhao, Y., M. Lv, H. Lin, Y. Cui, X. Wei, Y. Qin, K. Kohama, and Y. Gao. 2013. Rho-associated protein kinase isoforms stimulate proliferation of vascular smooth muscle cells through ERK and induction of cyclin D1 and PCNA. Biochem. Biophys. Res. Commun. 432:488-493. 